\title{
Mood, psychomotor, and cognitive function in major depressive disorder: from biomarkers to rapid-acting antidepressants
}

\author{
Kenji Hashimoto ${ }^{1}$
}

Published online: 27 August 2019

○) Springer-Verlag GmbH Germany, part of Springer Nature 2019

Patients with major depressive disorder (MDD) have psychomotor and cognitive impairments in addition to mood disorder. In this issue, Koo et al. [1] investigated the utility of combining biomarkers related to executive dysfunctions, motor activity, and neurophysiological patterns in patients with MDD $(n=20)$ and healthy control subjects $(n=20)$. Patients with MDD displayed significant impairments in executive functions and the reduction of daily motor activity. As measured by electroencephalogram (EEG) parameters (i.e., asymmetry in alpha power and reduction in alertness), patients with MDD showed increased right frontal lobe activity compared with left frontal lobe activity and lower brain arousal than that in healthy controls. Regression analysis showed that executive functions and alpha power asymmetry index in the EEG discriminated between patients with MDD and healthy controls with $78 \%$ accuracy. Furthermore, the interaction between motor activity and the EEG-vigilance stage alongside executive function increased the accuracy to $81 \%$. This preliminary study suggests that combining these biomarkers results in a reliable biomarker for MDD. However, in this study, the patients with MDD were medicated with antidepressants, such as selective serotonin reuptake inhibitor (SSRI) or serotonin norepinephrine reuptake inhibitor (SNRI), although the antidepressants were discontinued 3 days before the investigation. Further study using a large sample size of drug-naïve patients with MDD is needed.

Proactive control allows individuals to anticipate and prepare for upcoming events. In this issue, Hoffmann et al. [2] investigated cerebral blood flow responses in patients with MDD $(n=40)$ and healthy control subjects $(n=40)$ during a pre-cued anti-saccade task that required preparatory

Kenji Hashimoto

hashimoto@faculty.chiba-u.jp

1 Division of Clinical Neuroscience, Chiba University Center for Forensic Mental Health, 1-8-1 Inohana, Chiba 260-8670, Japan attention and proactive inhibition. Right dominant blood flow increased during pro-saccade and anti-saccade preparations. The increase was smaller in patients with MDD than in controls. Patients with MDD exhibited higher error rates than controls for anti-saccade, but not for pro-saccade tasks. This study suggests that there are impairments in proactive control in patients with MDD; however, further supportive studies are necessary.

Growing evidence suggests that vascular endothelial growth factor (VEGF) plays a role in the pathophysiology of MDD. A meta-analysis demonstrated that the blood levels of VEGF were significantly higher in patients with MDD than in healthy controls. This suggests that elevated blood VEGF levels are a disease biomarker for depression [3]. Despite the link between VEGF and MDD, there are few reports that show VEGF gene polymorphisms in MDD. A genome-wide association study showed four single nucleotide polymorphisms (SNPs) (i.e., rs4416670, rs6921438, rs6993770, and rs 10738760 ) which explain approximately $50 \%$ of the heritability of circulating VEGF levels [4]. In this issue, Nguyen et al. [5] investigated the relationship between brain volume and these four SNPs in the first-episode drug-naiive patients with MDD $(n=38)$ and healthy subjects $(n=39)$. There was a genotype-diagnosis interaction for rs6921438 in the subiculum of the left hippocampus. This preliminary study suggests a link between VEGF-related SNP rs6921438 and subiculum atrophy in first-episode drug-naïve patients with MDD. Further study using a large sample size of patients with MDD is needed to confirm the role of $V E G F$-related SNP rs6921438 in subiculum atrophy in MDD.

The discovery of the robust antidepressant actions of ketamine in treatment-resistant patients with MDD is a serendipity in the psychopharmacological treatment of depression $[6,7]$. However, precise molecular and cellular mechanisms underlying ketamine's antidepressant effects remain to be elucidated. Deyama et al. [8] recently reported the role of neuronal VEGF signaling in the prefrontal cortex on the rapid antidepressant effects of ketamine; however, further 
studies using rodents with depression-like phenotypes are needed [7]. On March 5, 2019, the United State Food Drug Administration approved the use of $(S)$-ketamine nasal spray for treatment-resistant depression. Due to the risk of serious adverse effects and the potential for abuse and misuse of the drug $(S)$-ketamine nasal spray is only available through a restricted distribution system under a Risk Evaluation and Mitigation Strategy (REMS). Recently, Yang et al. [9] reported that $(S)$-norketamine, a major metabolite of $(S)$ ketamine, elicits rapid and sustained antidepressant effects in rodent models of depression and has a potency similar to that of $(S)$-ketamine. Importantly, unlike $(S)$-ketamine, $(S)$-norketamine does not cause behavioral or biochemical abnormalities in rodents. Collectively $(S)$-norketamine could be a safer alternative antidepressant than $(S)$-ketamine and racemic ketamine for use in patients with $\operatorname{MDD}[7,9,10]$.

Acknowledgements This study was partly supported by AMED under Grant number JP19dm0107119.

\section{Compliance with ethical standards}

Conflict of interest Dr. Hashimoto is the inventor of filed patent applications on "The use of $R$-ketamine in the treatment of psychiatric diseases" and " $(S)$-norketamine and salt thereof as pharmaceutical" by the Chiba University.

\section{References}

1. Koo PC, Berger C, Kronenberg G, Bartz J, Wybitul P, Reis O, Hoeppner J (2019) Combined cognitive, psychomotor and electrophysiological biomarkers in major depressive disorder. Eur Arch Psychiatry Clin Neurosci. https://doi.org/10.1007/s0040 6-018-0952-9
2. Hoffmann A, Ettinger U, Montoro C, Reyes del Paso G, Duschek S (2019) Cerebral blood flow responses during prosaccade and antisaccade preparation in major depression. Eur Arch Psychiatry Clin Neurosci. https://doi.org/10.1007/s00406-018-0956-5

3. Tseng PT, Cheng YS, Chen YW, Wu CK, Lin PY (2015) Increased levels of vascular endothelial growth factor in patients with major depressive disorder: a meta-analysis. Eur Neuropsychopharmacol 25(10):1622-1630

4. Debette S, Visvikis-Siest S, Chen MH, Ndiaye NC, Song C, Destefano A, Safa R, Azimi Nezhad M, Sawyer D, Marteau JB, Xanthakis V, Siest G, Sullivan L, Pfister M, Smith H, Choi SH, Lamont J, Lind L, Yang Q, Fitzgerald P, Ingelsson E, Vasan RS, Seshadri S (2011) Identification of cis- and trans-acting genetic variants explaining up to half the variation in circulating vascular endothelial growth factor levels. Circ Res 109(5):554-563

5. Nguyen L, Kakeda S, Katsuki A, Sugimoto K, Otsuka Y, Ueda I, Igata R, Watanabe K, Kishi T, Iwata N, Korogi Y, Yoshimura R (2019) Relationship between VEGF-related gene polymorphisms and brain morphology in treatment-naïve patients with first-episode major depressive disorder. Eur Arch Psychiatry Clin Neurosci. https://doi.org/10.1007/s00406-018-0953-8

6. Krystal JH, Abdallah CG, Sanacora G, Charney DS, Duman RS (2019) Ketamine: a paradigm shift for depression research and treatment. Neuron 101(5):774-778

7. Hashimoto K (2019) Rapid-acting antidepressant ketamine, its metabolites and other candidates: a historical overview and future perspective. Psychiatry Clin Neurosci. https://doi.org/10.1111/ pcn. 12902

8. Deyama S, Bang E, Wohleb ES, Li XY, Kato T, Gerhard DM, Dutheil S, Dwyer JM, Taylor SR, Picciotto MR, Duman RS (2019) Role of neuronal VEGF signaling in the prefrontal cortex in the rapid antidepressant effects of ketamine. Am J Psychiatry 176(5):388-400

9. Yang C, Kobayashi S, Nakao K, Dong C, Han M, Qu Y, Ren Q, Zhang JC, Ma M, Toki H, Yamaguchi JI, Chaki S, Shirayama Y, Nakazawa K, Manabe T, Hashimoto K (2018) AMPA receptor activation-independent antidepressant actions of ketamine metabolite $(S)$-norketamine. Biol Psychiatry 84(8):591-600

10. Hashimoto K, Yang C (2019) Is (S)-norketamine an alternative antidepressant for esketamine? Eur Arch Psychiatry Clin Neurosci. https://doi.org/10.1007/s00406-018-0922-2 